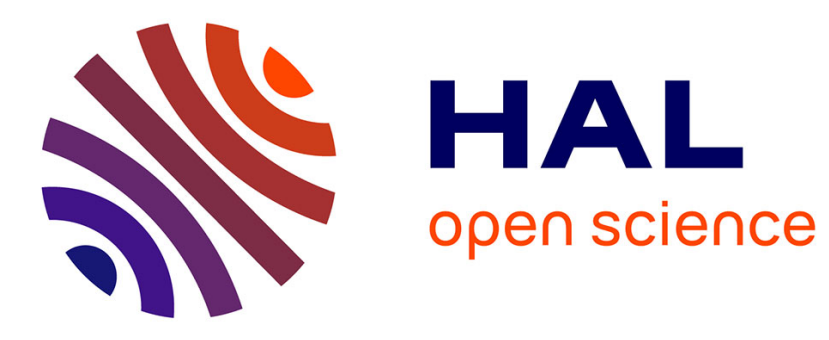

\title{
Asymptotically efficient GNSS trilateration
}

François Vincent, Eric Chaumette, Christophe Charbonnieras, Jonathan

Israel, Marion Aubault, Franck Barbiero

\section{To cite this version:}

François Vincent, Eric Chaumette, Christophe Charbonnieras, Jonathan Israel, Marion Aubault, et al.. Asymptotically efficient GNSS trilateration. Signal Processing, 2017, 133, pp.270-277. 10.1016/j.sigpro.2016.11.027 . hal-01428433

\section{HAL Id: hal-01428433 \\ https://hal.science/hal-01428433}

Submitted on 22 Oct 2020

HAL is a multi-disciplinary open access archive for the deposit and dissemination of scientific research documents, whether they are published or not. The documents may come from teaching and research institutions in France or abroad, or from public or private research centers.
L'archive ouverte pluridisciplinaire HAL, est destinée au dépôt et à la diffusion de documents scientifiques de niveau recherche, publiés ou non, émanant des établissements d'enseignement et de recherche français ou étrangers, des laboratoires publics ou privés. 


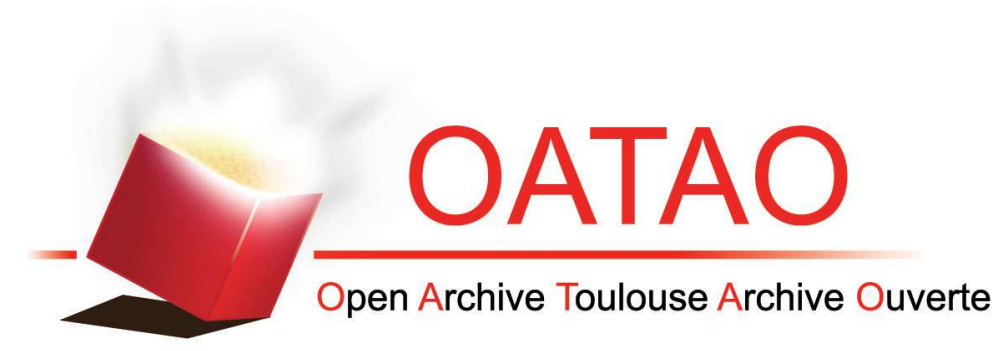

\section{Open Archive Toulouse Archive Ouverte (OATAO)}

OATAO is an open access repository that collects the work of some Toulouse researchers and makes it freely available over the web where possible.

This is an author's version published in: https://oatao.univ-toulouse.fr/26810

Official URL : https://doi.org/10.1016/j.sigpro.2016.11.027

\section{To cite this version :}

Vincent, François and Chaumette, Eric and Charbonnieras, Christophe and Israel, Jonathan and Aubault, Marion and Barbiero, Franck Asymptotically efficient GNSS trilateration. (2017) Signal Processing, 133. 270-277. ISSN 0165-1684

Any correspondence concerning this service should be sent to the repository administrator: tech-oatao@listes-diff.inp-toulouse.fr 


\title{
Asymptotically efficient GNSS trilateration
}

\author{
François Vincent $^{\mathrm{a}, *}$, Eric Chaumette ${ }^{\mathrm{a}}$, Christophe Charbonnieras $^{\mathrm{b}}$, Jonathan Israel $^{\mathrm{b}}$, \\ Marion Aubault ${ }^{\mathrm{c}}$, Franck Barbiero ${ }^{\mathrm{c}}$ \\ a University of Toulouse-ISAE, Department of Electronics, Optronics and Signal, 10 Avenue Edouard Belin, 31055, Toulouse, France \\ b ONERA, The French Aerospace Lab, DEMR/RCP, 2 avenue Edouard Belin, 31055 Toulouse CEDEX 4, France \\ c CNES, The French Space Agency, 18, av. Edouard Belin 31401 TOULOUSE Cedex 9, France
}

\section{A R T I C L E I N F O}

\section{Keywords:}

Approximate Maximum Likelihood estimation GNSS

GPS

Direct Position Estimation

EXIP

Trilateration

\begin{abstract}
A B S T R A C T
Localization based on the reception of radio-frequency waveforms is a crucial problem in many civilian or military applications. It is also the main objective of all Global Navigation Satellite System (GNSS). Given delayed and Doppler shifted replicas of the satellites transmitted signals, the most widespread approach consists in a suboptimal two-step procedure. First, estimate the delays and Dopplers from each satellite independently, then estimate the user position and speed thanks to a Least Square (LS) minimization. More accurate and robust techniques, such as a direct Maximum Likelihood (ML) maximization, that exploit the links in between the different channels exist but suffer from an heavy computational burden that prevent their use in real time applications. Two-steps procedures with an appropriate Weighted LS (WLS) minimization are shown to be asymptotically equivalent to the ML procedure. In this paper, we develop a closed-form expression of this WLS asymptotically efficient solution. We show that this simple expression is the sum of two terms. The first one, depending on the pseudo-ranges is the widespread used WLS solution. The second one is a Doppler-aided corrective term that should be taken into account to improve the position estimation when the observation time increases.
\end{abstract}

\section{Introduction}

The main objective of any Global Navigation Satellite System (GNSS) receiver is to estimate its position and speed from all in-view satellite signals. The common approach consists in a two step procedure. First, from each satellite signal, a propagation delay and a Doppler shift are calculated separately. Then, using these estimates, a trilateration step is performed to compute the receiver position and speed. The first stage is easily conducted by correlation of the received signal with the known orthogonal direct-sequence spread spectrum transmitted by each satellite. Depending on the knowledge available on the actual delay and Doppler, a complete (acquisition) or a local search (tracking) is conducted. The second step is a non-linear least-square minimization problem usually performed iteratively by linearising the cost function next to an initial guess. This guess can be computed using a Bancroft algorithm [1] for instance.

This widely used two-step procedure exhibits near optimal performances in open-sky environments but can dramatically degrade in case of complicated scenarios comprising jamming, multipath and channel fading [2]. These effects are known to be the more damageable for the localization precision as they are difficult to compensate by additional aids (such as differential measurements or assisted error modelling). In this case, a direct Maximum Likelihood (ML) position and speed estimation - computed from all in-view satellite signals - will outperform the two-step procedure. Indeed, this so-called Direct Position Estimation (DPE) exploits the fact that the ranges from the satellites are all calculated from the same point, the receiver position, and share a mutual information. The two-step scheme does not exploit this prior knowledge leading to possible incompatible delay estimations and consequently a wrong position calculation. Moreover the information provided by the Doppler shift is rarely exploited in the two-step procedure whereas it handles a useful knowledge on the user position. This piece of information could be very useful to improve the position estimation. It can be noticed that some former systems only exploited this Doppler information for positioning, just like the first global satellite navigation system: TRANSIT. The DPE, that intrinsically exploits this knowledge is known to be asymptotically efficient and unbiased. That is to say, the position and speed correlation error matrix tends toward the lower error bound given by the inverse of the Fisher Information Matrix (FIM). In the GNSS context, the FIM has been

\footnotetext{
* Corresponding author.

E-mail addresses: francois.vincent@isae.fr (F. Vincent), eric.chaumette@isae.fr (E. Chaumette), Christophe.Charbonnieras@onera.fr (C. Charbonnieras), Jonathan.Israel@onera.fr (J. Israel), Marion.Aubault@cnes.fr (M. Aubault), franck.barbiero@cnes.fr (F. Barbiero).
} 
calculated in [3] both for the DPE and the two-step approach. Thus the asymptotic precision gain using a DPE procedure instead of the conventional approach can be easily evaluated. The gain can reach one order of magnitude on the position precision in case of large satellite signal power differences or in multipath environments [3]. The DPE has been presented in [4] and extended to the case of an array antenna receiver in [5]. Directly considering position and speed through a single step procedure also allows to introduce any prior information in a natural framework as we work directly with the user position. It can be easily associated with an Inertial Measurement Unit (IMU) for instance. Hence, the DPE philosophy has also been extended to the Bayesian approach in [6,7].

Unfortunately, the price to be paid in using this optimal direct processing is an extensive computational cost that prevents its use in practice. Indeed, DPE requires solving a non-linear multidimensional optimization problem. Possible solutions can be followed to circumvent this problem. For instance, one solution consists in splitting the multidimensional optimization procedure in a number of recursive and simpler searches. The Expectation Maximization (EM) principle, initially introduced in the radio-communication community [8] is an example of such a technique that has been successfully used in the GNSS context. Its extension to antenna array receivers through the Space Alternating Generalized Expectation Maximization (SAGE) is thereby a solution of choice to mitigate multipath $[9,10]$. Nevertheless, this kind of methods still remains computationally heavy and difficult to be considered in real-time applications.

It has been shown that the two-step procedure cannot outperform the DPE, but when choosing an appropriate weighted matrix for the WLS second step, the two solutions are asymptotically equivalent. The relevant weighted matrix corresponds to the Fisher Information Matrix (FIM) of the model at hand, calculated for the pseudo-ranges and Doppler frequencies. The explanation is linked to the so-called EXtended Invariance Principle (EXIP). EXIP was first introduced in [11] and is based on a re-parametrisation of the problem at hand leading to a less accurate formulation and a simpler solution. Then, these intermediate estimates can be refined to achieve asymptotically the performance of the initial model using an appropriate Weighted Least Square (WLS) minimization. This technique has been used in radar array processing $[12,13]$ for instance. The main idea behind EXIP is to find a reparametrisation that simplify the ML criterion to be maximized. The efficiency property of the ML is maintained (at least asymptotically) during the refining WLS step by using a matched weighting matrix. In the GNSS context, Antreich et al. have exploited the EXIP principle to simplify the joined Direction of Arrival (DoA), delay and Doppler estimation problem in relaxing the constraints on the steering vector structure of the array antenna [14]. In case of a single antenna receiver, it seems natural to re-parametrise the position and speed estimation issue into a delay and Doppler estimation problem assuming an independent processing for each satellite. Then, this approach gives a theoretical grounding to find an asymptotically efficient position and speed estimation thanks to the appropriate EXIP WLS procedure. This approach has been proposed by Closas et al. [2] in the GNSS context, and by Amar and Weiss for the reciprocal problem of active transmitter localization from a set of receivers [1518]. In all these papers, EXIP has mostly been used to compare the classical two-step procedure with the DPE concept but, to the best of our knowledge, no closed-from formulation of this EXIP DPE solution has been proposed.

In this paper, we propose to calculate this asymptotically efficient solution based on EXIP. The first step of the procedure remains the same as the classical delay and Doppler estimation and can be conducted both by an acquisition procedure or tracking loops. Hence, the classical GNSS receiver architecture does not have to be modified and any advanced tracking processing (Narrow Correlator [19], MEDLL [20],...) can be kept to improve this first stage. Then, we show that we can obtain a closed-form formulation of the FIM leading to a simple WLS solution. Moreover, we simplify this solution to show that it can be seen as the sum of two terms. The first one that only depends on the pseudo-ranges, is the widespread used WLS solution. The second one is Doppler based corrective term. This second term balance, in an optimal way, the information provided by the Doppler to improve the user position. This Doppler correction is negligible when the observation time is short, but can provide useful information if not. This dependence has been exploited in the literature, for instance by $\mathrm{Li}$ et al. in [21] and we give here, a theoretical framework to the Doppleraided positioning estimations.

The paper is organized as follows. Section 2 introduces the framework at hand and defines the signal model to be used. Then we make use of the EXIP to compute the optimal weight matrix in Section 3. Then, we simplify this solution in Section 4 to give an insightful interpretation. Numerical illustrations are provided in Sections 5 and 6 draws conclusions.

\section{Data model}

We assume that $K$ scaled, delayed and Doppler-shifted front waves, transmitted by each in-view satellite impinge on a GNSS receiver antenna. Under the narrowband assumption, the complex baseband model can be written as follows:

$x(t)=\sum_{k=0}^{K-1} \alpha_{k} \cdot c_{k}\left(t-\tau_{k}\right) \cdot e^{2 i \pi f_{k} t}+n(t)$

where

- $\alpha_{k}$ denotes each complex satellite signal amplitude, supposed to be deterministic and unknown,

- $c_{k}(t)$ stands for the transmitted complex baseband navigation signal spread by the pseudo-random code corresponding to the $k$-th satellite,

- $f_{0}$ is the carrier frequency,

- $n(t)$ corresponds to an additive zero-mean white Gaussian noise with variance $\sigma^{2}$,

- and $\tau_{k}, f_{k}$ are respectively the delay and Doppler frequency shift of the $k$-th satellite signal, observed from the receiver.

We suppose that $N$ snapshots are sampled at a $F_{s}=\frac{1}{T s}$ rate from $x(t)$, so that we can write:

$x=A \alpha+n$

where

- $\boldsymbol{x}=\left[x(0) \ldots x\left((N-1) T_{s}\right)\right]^{T}$,

- $\boldsymbol{A}=\left[\boldsymbol{a}_{0} \ldots \boldsymbol{a}_{K-1}\right]$ is the manifold corresponding to all in-view satellite signals, with $\boldsymbol{a}_{k}=\left[c_{k}\left(-\tau_{k}\right) \ldots c_{k}\left((N-1) T_{s}-\tau_{k}\right) \cdot e^{-2 i \pi f_{0} b_{k}(N-1) T_{s}}\right]^{T}$,

- $\boldsymbol{\alpha}=\left[\alpha_{0} \ldots \alpha_{K-1}\right]^{T}$ and,

- $\boldsymbol{n}=\left[n(0) \ldots n\left((N-1) T_{s}\right)\right]^{T}$

with.$^{T}$ being the transpose operation.

The observed delay $\tau_{k}$ and delay drift $b_{k}=-\frac{f_{k}}{f_{0}}$ depends on the actual relative distance and velocity from the satellite $k$ to the receiver, as well as secondary propagation effects (atmospheric and ionospheric additional delays,...) and receiver or transmitter defaults (clock bias and drift). They can be expressed as follows:

$\tau_{k} \simeq \frac{\left\|\boldsymbol{p}_{k}-\boldsymbol{p}\right\|}{c}+\tau_{0}+\delta \tau_{k} b_{k} \simeq \frac{\left(\boldsymbol{v}_{k}-\boldsymbol{v}\right)^{T} \cdot \boldsymbol{u}_{k}}{c}+b_{0}+\delta b_{k}$

where:

- $\quad \boldsymbol{p}, \boldsymbol{v}, \boldsymbol{p}_{k}$ and $\boldsymbol{v}_{k} \in \mathfrak{R}^{3}$ are respectively the position and velocity vectors of the receiver and of the $k$-th satellite, 
- $\boldsymbol{u}_{k}=\frac{\boldsymbol{p}_{k}-\boldsymbol{p}}{\left\|\boldsymbol{p}_{k}-\boldsymbol{p}\right\|}$ is the unitary vector toward the $k$-th satellite,

- $\tau_{0}$ and $b_{0}$ are the receiver clock delay and delay drift with respect to (w.r.t) the GPS time reference,

- $\delta \tau_{k}$ and $\delta b_{k}$ comprise all secondary biases (satellites clock defaults,...) and are supposed to be known from the navigation message.

Due to the non-linearity of the delay expression w.r.t. the receiver position, it is usual to consider a known initial position $\boldsymbol{p}_{0}$ and to linearise Eq. (3) for small position changes $\Delta \boldsymbol{p}=\boldsymbol{p}-\boldsymbol{p}_{0}$.

Thereby, the GNSS localization problem to be solved consists in estimating $\boldsymbol{\theta}=\left[\begin{array}{llll}\Delta \boldsymbol{p}^{T} & \boldsymbol{v}^{T} & \tau_{0} & b_{0}\end{array}\right]^{T}$ from the vector data $\boldsymbol{x}=\boldsymbol{A}(\boldsymbol{\theta}) \boldsymbol{\alpha}+\boldsymbol{n}$.

If we assume the complex amplitudes $\boldsymbol{\alpha}$ as deterministic and unknown, it is straightforward to show that the ML solution of the problem (also known as DPE) is given by maximizing the non-linear following criterion [4]:

$\widehat{\boldsymbol{\theta}}_{M L}=\operatorname{ArgMax}\left[\boldsymbol{x}^{H} \boldsymbol{P}_{\boldsymbol{A}}(\boldsymbol{\theta}) \boldsymbol{x}\right]$

where ${ }^{H}$ stands for the Hermitian transpose operation and $\boldsymbol{P}_{\boldsymbol{A}}=\boldsymbol{A}\left(\boldsymbol{A}^{H} \boldsymbol{A}\right)^{-1} \boldsymbol{A}^{H}$ is the projection matrix onto the signal subspace, spanned by the $K$ received signals. Essentially the DPE consists in searching for the best combination for $\boldsymbol{\theta}$ that maximise the norm of the projection of the data onto the signal subspace.

We can observe that $\boldsymbol{A}^{H} \boldsymbol{A} \simeq N$. $\boldsymbol{I}$, as the pseudo-random codes are almost orthogonal and the Doppler shift modulations are relatively slow compared to the signal variations. We can notice that this near orthogonality of the columns of $\boldsymbol{A}$ is the basis assumption that allows a separate processing for each satellite signal in all standard GNSS receiver. Thereby, we can simply write:

$\hat{\boldsymbol{\theta}}_{M L} \simeq \operatorname{ArgMax}\left[\left\|\boldsymbol{A}(\boldsymbol{\theta})^{H} \boldsymbol{x}\right\|^{2}\right]=\operatorname{ArgMax}\left[\sum_{k=0}^{K-1}\left|\boldsymbol{a}_{k}(\boldsymbol{\theta})^{H} \boldsymbol{x}\right|^{2}\right]=\operatorname{ArgMax}\left[C_{\theta}(\boldsymbol{\theta})\right]$

which is a non-linear 8-dimension optimization problem leading to a huge computational burden.

\section{Asymptotically efficient solution}

\subsection{EXIP formulation for the navigation problem}

In order to simplify this maximization while keeping asymptotically the efficiency properties of the ML, we make use of the EXIP principle [11]. The main idea behind EXIP is a re-parametrization of the problem at hand. As its name suggests, EXIP is an extension of the well-known invariance property of the ML when the re-parametrization is not bijective. More precisely, the objective is to find a mapping from $\boldsymbol{\theta} \in D_{\theta}$ to $\boldsymbol{\eta}=g(\boldsymbol{\theta})$ leading to a computationally simpler maximization. The point is to look for a solution in a larger space than the image space of $D_{\theta}$ by $g\left(g\left(D_{\theta}\right)\right.$ is a subspace of $D_{\eta}$ that constrains the solution):

$\widehat{\boldsymbol{\eta}}=\underset{\boldsymbol{\eta} \in D_{\eta}}{\operatorname{ArgMax}}\left[C_{\eta}(\boldsymbol{\eta})\right]$

where $C_{\eta}(g(\boldsymbol{\theta}))=C_{\theta}(\boldsymbol{\theta})$

Then, the following WLS estimate of $\boldsymbol{\theta}$ is shown to be asymptotically equivalent to $\widehat{\boldsymbol{\theta}}_{M L}[11]$

$\widehat{\boldsymbol{\theta}}=\underset{\boldsymbol{\theta} \in D_{\theta}}{\operatorname{ArgMin}}[\hat{\boldsymbol{\eta}}-g(\boldsymbol{\theta})]^{T} \boldsymbol{W}[\hat{\boldsymbol{\eta}}-g(\boldsymbol{\theta})]$

where $\boldsymbol{W}=-E\left\{\frac{\partial^{2}\left\|\boldsymbol{A}(\boldsymbol{\eta})^{H} \boldsymbol{x}\right\|^{2}}{\partial \boldsymbol{\eta} \partial \eta^{T}}\right\}_{\mid \boldsymbol{\eta}=\hat{\boldsymbol{\eta}}}$.

The key point of this procedure is to find a mapping leading to a simpler 2-steps procedure than the original one, in relaxing the original constraints. In the GNSS context, the re-parametrization that naturally comes in mind is to use each satellite delay and delay drift (or Doppler equally) instead of $\boldsymbol{\theta}$. Indeed, this mapping allows to relax the relationship that exists between the different pseudo-range measure- ments and allows an independent satellite by satellite processing during a first stage. Lets call $g$ this mapping function:

$g: \mathfrak{R}^{8} \longrightarrow \mathfrak{R}^{2 K} \boldsymbol{\theta} \longrightarrow \boldsymbol{\eta}=\left[\boldsymbol{\eta}_{0} \cdots \boldsymbol{\eta}_{K-1}\right]^{T}=g(\boldsymbol{\theta})$

with $\boldsymbol{\eta}_{k}=\left[\tau_{k} b_{k}\right]^{T}=g_{k}(\boldsymbol{\theta})$, according to Eq. (3).

We can notice that $D_{\eta}=\mathfrak{R}^{2 K}$ is larger than the space spanned by $g(\boldsymbol{\theta})$ for all possible $\boldsymbol{\theta} \in D_{\boldsymbol{\theta}}$. Working in this larger space is the key trick of the two-step processing leading to a simplified procedure, while relaxing the relations between the channels. The solution that maximises the ML criterion in this larger research space is given by:

$\widehat{\boldsymbol{\eta}}=\operatorname{ArgMax}\left[\left\|\boldsymbol{A}(\boldsymbol{\eta})^{H} \boldsymbol{x}\right\|^{2}\right]=\operatorname{ArgMax}\left[\sum_{k=0}^{K-1}\left|\boldsymbol{a}_{k}(\boldsymbol{\eta})^{H} \boldsymbol{x}\right|^{2}\right]$

or more simply, thanks to the independence provided by the reparametrization:

$\widehat{\boldsymbol{\eta}}_{k}=\operatorname{ArgMax}\left[\left|\boldsymbol{a}_{k}\left(\boldsymbol{\eta}_{k}\right)^{H} \boldsymbol{x}\right|^{2}\right] \quad k=0, \cdots(K-1)$

which corresponds to a correlation maximization with each satellite spread-spectrum sequence delayed and Doppler shifted. It is the usual correlation step for any GNSS receiver processing. It can be noticed that the proposed procedure is also compatible with IMU-aided or advanced processing techniques aiming at estimating or tracking more precisely delay and Doppler frequency.

Now, the second stage of the EXIP provides an asymptotically efficient estimate of $\widehat{\boldsymbol{\theta}}_{M L}$ as:

$\widehat{\boldsymbol{\theta}}_{\text {EXIP }}=\operatorname{ArgMin}\left[(\widehat{\boldsymbol{\eta}}-g(\boldsymbol{\theta}))^{T} \boldsymbol{W}(\widehat{\boldsymbol{\eta}}-g(\boldsymbol{\theta}))\right]$

where $\boldsymbol{W}$ is the FIM of the criterion to be minimized in Eq. (9), as stated in Eq. (7). The features of this criterion implies a block-diagonal structure for $\boldsymbol{W}$, as the cross derivatives are nulls, so that:

$\widehat{\boldsymbol{\theta}}_{\text {EXIP }}=\operatorname{ArgMin}\left[\sum_{k=0}^{K-1}\left(\widehat{\boldsymbol{\eta}}_{k}-g_{k}(\boldsymbol{\theta})\right)^{T} \boldsymbol{W}_{k}\left(\widehat{\boldsymbol{\eta}}_{k}-g_{k}(\boldsymbol{\theta})\right)\right]$

where $\boldsymbol{W}_{k}=-E\left\{\frac{\partial^{2}\left|a_{k}\left(\boldsymbol{\eta}_{k}\right)^{H} \boldsymbol{x}\right|^{2}}{\partial \boldsymbol{\eta}_{k} \partial \boldsymbol{\eta}_{k}^{T}}\right\}_{\mid \boldsymbol{\eta}_{k}=\hat{\eta}_{k}}$.

\subsection{Weight computation}

Let's start with $\boldsymbol{W}_{k}$ calculation. $\boldsymbol{W}_{k}$ can be easily reformulated as follows:

$\boldsymbol{W}_{k}=-\frac{\partial^{2}\left[\boldsymbol{a}_{k}\left(\boldsymbol{\eta}_{k}\right)^{H} \boldsymbol{R} \boldsymbol{a}_{k}\left(\boldsymbol{\eta}_{k}\right)\right]}{\partial \boldsymbol{\eta}_{k} \partial \boldsymbol{\eta}_{k}^{T} \quad}$

with $\boldsymbol{R}=\mathcal{E}\left\{\boldsymbol{x} \boldsymbol{x}^{H}\right\}=\boldsymbol{A} \boldsymbol{R}_{\boldsymbol{\alpha}} \boldsymbol{A}^{H}+\sigma^{2} \boldsymbol{I}$, where $\boldsymbol{R}_{\boldsymbol{\alpha}}=\mathcal{E}\left\{\boldsymbol{\alpha} \boldsymbol{\alpha}^{H}\right\}$ is the covariance matrix of the sources amplitudes, supposed to be diagonal.

In order to clearly bring out the influence of the delay and Doppler, we write down $\boldsymbol{a}_{k}\left(\boldsymbol{\eta}_{k}\right)=\boldsymbol{c}_{k}\left(\tau_{k}\right) \odot \boldsymbol{e}_{k}\left(b_{k}\right)$ where $\odot$ denotes the Hadamard product, $\boldsymbol{c}_{k}\left(\tau_{k}\right)=\left[c_{k}\left(-\tau_{k}\right) \cdots c_{k}\left((N-1) T_{s}-\tau_{k}\right)\right]^{T}$ is the $k$-th code signal and $\boldsymbol{e}_{k}\left(b_{k}\right)=\left[1 \cdots e^{-2 i \pi f_{0} b_{k}(N-1) T_{s}}\right]^{T}$ the associated Doppler shift.

The four elements of $\boldsymbol{W}_{k}$ can then be expressed as:

$\boldsymbol{W}_{k_{1,1}}=-2 \operatorname{Re}\left[\left(\partial^{2} \boldsymbol{c}_{k} \odot \boldsymbol{e}_{k}\right)^{H} \boldsymbol{R} \boldsymbol{a}_{k}+\left(\partial \boldsymbol{c}_{k} \odot \boldsymbol{e}_{k}\right)^{H} \boldsymbol{R}\left(\partial \boldsymbol{c}_{k} \odot \boldsymbol{e}_{k}\right)\right]$

$\boldsymbol{W}_{k_{1,2}}=-2 \operatorname{Re}\left[\left(-2 i \pi f_{0}\right)\left(\boldsymbol{t} \odot \partial \boldsymbol{c}_{k} \odot \boldsymbol{e}_{k}\right)^{H} \boldsymbol{R} \boldsymbol{a}_{k}+\left(2 i \pi f_{0}\right)\left(\partial \boldsymbol{c}_{k} \odot \boldsymbol{e}_{k}\right)^{H} \boldsymbol{R}\left(\boldsymbol{t} \odot \boldsymbol{a}_{k}\right)\right]$

$=\boldsymbol{W}_{k_{2,1}}^{*} \boldsymbol{W}_{k_{2,2}}=-2 \operatorname{Re}\left[-\left(2 \pi f_{0}\right)^{2}\left(\boldsymbol{t}^{\mathbf{2}} \odot \boldsymbol{a}_{k}\right)^{H} \boldsymbol{R} \boldsymbol{a}_{k}\right.$

$\left.+\left(2 \pi f_{0}\right)^{2}\left(\boldsymbol{t} \odot \boldsymbol{a}_{k}\right)^{H} \boldsymbol{R}\left(\boldsymbol{t} \odot \boldsymbol{a}_{k}\right)\right]$

where $\quad \partial c_{k}=\frac{\partial c_{k}}{\partial t}\left(t-\tau_{k}\right), \quad \partial^{2} c_{k}=\frac{\partial^{2} c_{k}}{\partial t^{2}}\left(t-\tau_{k}\right), \quad t=\left[0 \cdots(N-1) T_{s}\right]^{T} \quad$ and $\boldsymbol{t}^{2}=\left[0 \cdots(N-1)^{2} T_{s}^{2}\right]^{T}$.

Now using the results in the Appendix A, it is straightforward to show that: 
$\boldsymbol{W}_{k}=P_{k}\left(\frac{2}{3} \pi^{2} N^{2}\right)\left[\begin{array}{cc}B^{2} & 0 \\ 0 & f_{0}^{2} T_{c}^{2}\end{array}\right] \stackrel{\Delta}{=} P_{k} \boldsymbol{B}$

where $P_{k}=\mathcal{E}\left\{\left|\alpha_{k}\right|^{2}\right\}$ is the power received from the $k$-th satellite signal and $T_{c}=N T_{s}$ is the integration time.

\subsection{WLS closed-form solution}

As it is usually done in every GNSS receiver, we first linearise Eq. (3) around the guess position $\boldsymbol{p}_{0}$.

$\tau_{k} \simeq \frac{d_{k_{0}}}{c}-\frac{\boldsymbol{u}_{k_{0}}^{T} \boldsymbol{\Delta} \boldsymbol{p}}{c}+\tau_{0}+\delta \tau_{k} b_{k} \simeq \frac{\boldsymbol{v}_{k}^{T} \cdot \boldsymbol{u}_{k_{0}}}{c}-\frac{\boldsymbol{u}_{k_{0}}^{T} \cdot \boldsymbol{v}}{c}-\frac{\boldsymbol{v}_{k}^{T} \boldsymbol{P}_{k_{0}}^{\perp} \boldsymbol{\Delta} \boldsymbol{p}}{c d_{k_{0}}}+b_{0}$

$+\delta b_{k}$

where $\boldsymbol{u}_{k_{0}}=\frac{\boldsymbol{p}_{k}-\boldsymbol{p}_{0}}{\left\|\boldsymbol{p}_{k}-\boldsymbol{p}_{0}\right\|}$ is the supposed direction toward the $k$-th satellite, $\boldsymbol{P}_{k_{0}}^{\perp}=\boldsymbol{I}-\boldsymbol{u}_{k_{0}} \boldsymbol{u}_{k_{0}}^{H}$ is the projection onto the subspace orthogonal to $\boldsymbol{u}_{k_{0}}$ and $d_{k_{0}}=\left\|\boldsymbol{p}_{k}-\boldsymbol{p}_{0}\right\|$ is the supposed distance to the $k$-th satellite. In order to simplify the calculation to come, we split the unknown vector $\boldsymbol{\theta}$ into the mutual biases vector $\boldsymbol{\beta}=\left[\tau_{0} b_{0}\right]^{T}$ and $\boldsymbol{\phi}=\frac{1}{c}\left[\boldsymbol{\Delta} \boldsymbol{p}^{T} \boldsymbol{v}^{T}\right]^{T}$, whose influence differs for each satellite. Thus, we can re-write Eq. (16) as:

$\boldsymbol{\eta}_{k}-\boldsymbol{\beta}_{k}=\boldsymbol{\beta}+\boldsymbol{H}_{k} \boldsymbol{\phi}$

where $\boldsymbol{\beta}_{k}=\left[\left(\frac{d_{k_{0}}}{c}+\delta \tau_{k}\right)\left(\frac{v_{k}^{T} \cdot u_{k_{0}}}{c}+\delta b_{k}\right)\right]^{T}$ comprises all known biases and $\boldsymbol{H}_{k}=-\left[\begin{array}{cc}\boldsymbol{u}_{k_{0}}^{T} & 0^{T} \\ \boldsymbol{\nu}_{k_{0}}^{T} & \boldsymbol{u}_{k_{0}}^{T}\end{array}\right]$

with $\boldsymbol{\nu}_{k_{0}}=\frac{\boldsymbol{P}_{k_{0}}^{\perp} v_{k}}{d_{k_{0}}}$ which describes the angular velocity of the $k$ th satellite when assuming a static receiver and where $\mathbf{0}=\left[\begin{array}{lll}0 & 0 & 0\end{array}\right]^{T}$.

In order to determine the $\boldsymbol{\beta}$ vector that minimises the WLS problem stated in Eq. (12), we have to derive the criterion w.r.t to $\boldsymbol{\beta}$ and equal the result to zero. It is straightforward to obtain:

$\widehat{\boldsymbol{\beta}}_{E X I P}=\left(\sum_{k=0}^{K-1} \boldsymbol{W}_{k}\right)^{-1}\left(\sum_{k=0}^{K-1} \boldsymbol{W}_{k}\left(\Delta \boldsymbol{\eta}_{k}-\boldsymbol{H}_{k} \boldsymbol{\phi}\right)\right)$

where $\Delta \boldsymbol{\eta}_{k} \stackrel{\Delta}{=} \widehat{\boldsymbol{\eta}}_{k}-\boldsymbol{\beta}_{k}$.

Inserting the result from Eq. (15), we have the following simple expression for the receiver clock biases

$\widehat{\boldsymbol{\beta}}_{E X I P}=\bar{\Delta} \boldsymbol{\eta}-\overline{\boldsymbol{H}} \boldsymbol{\phi}$

where $\overline{\boldsymbol{\Delta} \boldsymbol{\eta}}=\frac{\sum_{k=1}^{K-1} P_{k} \boldsymbol{\Delta} \boldsymbol{\eta}_{k}}{\sum_{k=1}^{K-1} P_{k}}$ and $\overline{\boldsymbol{H}}=\frac{\sum_{k=1}^{K-1} P_{k} \boldsymbol{H}_{k}}{\sum_{k=1}^{K-1} P_{k}}$ are the weighted-means of $\boldsymbol{\Delta} \boldsymbol{\eta}_{k}$ and $\boldsymbol{H}_{k}$ associated with the power of each channel. Replacing this result into Eq. (12) yields the following criterion to be minimized w.r.t to $\phi$ :

$\sum_{k=1}^{K-1}\left[\left(\boldsymbol{\Delta} \boldsymbol{\eta}_{k}-\overline{\boldsymbol{\Delta} \boldsymbol{\eta}}\right)-\left(\boldsymbol{H}_{k}-\overline{\boldsymbol{H}}\right) \boldsymbol{\phi}\right]^{T} \boldsymbol{W}_{k}\left[\left(\boldsymbol{\Delta} \boldsymbol{\eta}_{k}-\overline{\boldsymbol{\Delta} \boldsymbol{\eta}}\right)-\left(\boldsymbol{H}_{k}-\overline{\boldsymbol{H}}\right) \boldsymbol{\phi}\right]$

whose solution is

$\widehat{\boldsymbol{\phi}}_{E X I P}=\left[\sum_{k=1}^{K-1}\left(\boldsymbol{H}_{k}-\overline{\boldsymbol{H}}\right)^{T} \boldsymbol{W}_{k}\left(\boldsymbol{H}_{k}-\overline{\boldsymbol{H}}\right)\right]^{-1} \times\left[\sum_{k=1}^{K-1}\left(\boldsymbol{H}_{k}-\overline{\boldsymbol{H}}\right)^{T} \boldsymbol{W}_{k}\left(\boldsymbol{\Delta} \boldsymbol{\eta}_{k}-\overline{\boldsymbol{\Delta} \boldsymbol{\eta})}\right]\right.$

which gives the asymptotically optimum position and speed of the receiver from the delays and Doppler observed from all in-view satellites. We can notice that these optimum estimates depend on the power of each signal, $P_{\boldsymbol{k}}$ (through $\boldsymbol{W}_{k}, \overline{\boldsymbol{H}}$ and $\bar{\Delta} \boldsymbol{\eta}$ ) that can be easily estimated from EXIP first step as $\widehat{\boldsymbol{\alpha}}=\left(\boldsymbol{A}(\widehat{\boldsymbol{\eta}})^{H} \boldsymbol{A}(\widehat{\boldsymbol{\eta}})\right)^{-1} \boldsymbol{A}(\widehat{\boldsymbol{\eta}})^{H} \boldsymbol{x}$ and $\widehat{P}_{k}=\widehat{\boldsymbol{\alpha}}^{2}$. We can also compute the optimum estimates of the receiver clock biases as $\widehat{\boldsymbol{\beta}}=\overline{\Delta \boldsymbol{\eta}}-\overline{\boldsymbol{H}} \widehat{\boldsymbol{\phi}}_{E X I P}$.

\section{4. . Insights and comments}

The solution from Eq. (22) requires a $6 \times 6$ matrix inversion, and we propose to simplify this expression to give a meaningful interpretation. Let's introduce:

- $\boldsymbol{U}=\left[\sqrt{P_{0}}\left(\boldsymbol{u}_{0_{0}}-\overline{\boldsymbol{u}}\right) \cdots \sqrt{P_{K-1}}\left(\boldsymbol{u}_{(K-1)_{0}}-\overline{\boldsymbol{u}}\right)\right]$ where $\overline{\boldsymbol{u}}=\frac{\sum_{k=1}^{K-1} P_{k} \boldsymbol{u}_{k}}{\sum_{k=1}^{K-1} P_{k}}$ can be viewed as a weighted mean satellite direction,

- $\boldsymbol{V}=\left[\sqrt{P_{0}}\left(\nu_{0_{0}}-\bar{\nu}\right) \cdots \sqrt{P_{K-1}}\left(\nu_{(K-1)_{0}}-\bar{\nu}\right)\right]$ where $\bar{\nu}=\frac{\sum_{k=1}^{K-1} P_{k} \nu_{k}}{\sum_{k=1}^{K-1} P_{k}}$,

- $\Delta \boldsymbol{\tau}=\left[\underline{\sqrt{P_{0}}}\left(\Delta \tau_{0}-\overline{\Delta \tau}\right) \cdots \sqrt{P_{K-1}}\left(\Delta \tau_{K-1}-\overline{\Delta \tau}\right)\right]^{T}$ where $\Delta \tau_{k}=\Delta \boldsymbol{\eta}_{k}(1)$ and $\overline{\Delta \tau}=\bar{\Delta} \boldsymbol{\eta}(1)$,

- $\boldsymbol{\Delta}_{-}=\left[\sqrt{P_{0}}\left(\Delta b_{0}-\overline{\Delta b}\right) \cdots \sqrt{P_{K-1}}\left(\Delta b_{K-1}-\overline{\Delta b}\right)\right]^{T}$ where $\Delta b_{k}=\boldsymbol{\Delta} \boldsymbol{\eta}_{k}(2)$ and $\overline{\Delta b}=\bar{\Delta} \boldsymbol{\eta}(2)$.

In this form, the matrix and vector that contribute to Eq. (22) can be expressed simply as follows:

$\left[\sum_{k=1}^{K-1}\left(\boldsymbol{H}_{k}-\overline{\boldsymbol{H}}\right)^{T} \boldsymbol{W}_{k}\left(\boldsymbol{H}_{k}-\overline{\boldsymbol{H}}\right)\right]=\boldsymbol{B}(2,2)\left[\begin{array}{cc}\left(r \boldsymbol{U} \boldsymbol{U}^{T}+\boldsymbol{V} \boldsymbol{V}^{T}\right) & \left(\boldsymbol{V} \boldsymbol{U}^{T}\right) \\ \left(\boldsymbol{U} \boldsymbol{V}^{T}\right) & \left(\boldsymbol{U} \boldsymbol{U}^{T}\right)\end{array}\right]$

and

$\left[\sum_{k=1}^{K-1}\left(\boldsymbol{H}_{k}-\overline{\boldsymbol{H}}^{T} \boldsymbol{W}_{k}\left(\boldsymbol{\Delta} \boldsymbol{\eta}_{k}-\overline{\boldsymbol{\Delta} \boldsymbol{\eta})}\right]=-\boldsymbol{B}(2,2)\left[\begin{array}{c}r \boldsymbol{U} \boldsymbol{\Delta} \boldsymbol{\tau}+\boldsymbol{V} \boldsymbol{\Delta} \boldsymbol{b} \\ \boldsymbol{U} \boldsymbol{\Delta} \boldsymbol{b}\end{array}\right]\right.\right.$

where $\boldsymbol{B}$ is the matrix defined in Eq. (15) and $r=\frac{\boldsymbol{B}(1,1)}{\boldsymbol{B}(2,2)}=\left(\frac{B}{f_{0} T_{c}}\right)^{2}$.

Now using the matrix inversion formula in block form, it is straightforward to obtain:

$$
\begin{aligned}
& {\left[\sum_{k=1}^{K-1}\left(\boldsymbol{H}_{k}-\overline{\boldsymbol{H}}\right)^{T} \boldsymbol{W}_{k}\left(\boldsymbol{H}_{k}-\overline{\boldsymbol{H}}\right)\right]^{-1}} \\
& \quad=\boldsymbol{B}(2,2)^{-1}\left[\begin{array}{cc}
\boldsymbol{\Omega}^{-1} & -\boldsymbol{\Omega}^{-1} \boldsymbol{V} \boldsymbol{U}^{T}\left(\boldsymbol{U} \boldsymbol{U}^{T}\right)^{-1} \\
-\left(\boldsymbol{U} \boldsymbol{U}^{T}\right)^{-1} \boldsymbol{U} \boldsymbol{V}^{T} \boldsymbol{\Omega}^{-1} & \left(\boldsymbol{U} \boldsymbol{U}^{T}\right)^{-1}+\left(\boldsymbol{U} \boldsymbol{U}^{T}\right)^{-1} \boldsymbol{U} \boldsymbol{V}^{T} \boldsymbol{\Omega}^{-1} \boldsymbol{V} \boldsymbol{U}^{T}\left(\boldsymbol{U} \boldsymbol{U}^{T}\right)^{-1}
\end{array}\right]
\end{aligned}
$$

with

$\boldsymbol{\Omega}=\left[r \boldsymbol{U} \boldsymbol{U}^{T}+\boldsymbol{V} \boldsymbol{V}^{T}-\boldsymbol{V} \boldsymbol{U}^{T}\left(\boldsymbol{U} \boldsymbol{U}^{T}\right)^{-1} \boldsymbol{U} \boldsymbol{V}^{T}\right]=\left[r \boldsymbol{U} \boldsymbol{U}^{T}+\boldsymbol{V} \boldsymbol{P}_{U^{T}}^{\perp} \boldsymbol{V}^{T}\right]$

where $\boldsymbol{P}_{U^{T}}^{\perp}$ is the projection onto the subspace orthogonal to the subspace spanned by the 3 columns of $\boldsymbol{U}^{T}$.

From Eqs. (24) and (25), it is straightforward to obtain a closedform expression for the receiver position and velocity. As the main objective of many GNSS receiver is to estimate its position, let's focus on the 3 first components of $\widehat{\boldsymbol{\phi}}_{E X I P}$ corresponding to $\widehat{\boldsymbol{p}}_{E X I P}$ :

$\frac{1}{c}\left(\widehat{\boldsymbol{p}}_{E X I P}-\boldsymbol{p}_{0}\right)=-\boldsymbol{\Omega}^{-1}(r \boldsymbol{U} \boldsymbol{\Delta} \boldsymbol{\tau}+\boldsymbol{V} \boldsymbol{\Delta} \boldsymbol{b})-\boldsymbol{\Omega}^{-1} \boldsymbol{V} \boldsymbol{U}^{T}\left(\boldsymbol{U} \boldsymbol{U}^{T}\right)^{-1} \boldsymbol{U} \boldsymbol{\Delta} \boldsymbol{b}$

or equivalently,

$\widehat{\boldsymbol{p}}_{E X I P}=\boldsymbol{p}_{0}-c\left[r \boldsymbol{U} \boldsymbol{U}^{T}+\boldsymbol{V} \boldsymbol{P}_{U^{T}}^{\perp} \boldsymbol{V}^{T}\right]^{-1} \times\left(r \boldsymbol{U} \boldsymbol{\Delta} \boldsymbol{\tau}+\boldsymbol{V} \boldsymbol{P}_{U^{T}}^{\perp} \boldsymbol{\Delta b}\right)$

(28) provides a simple formulation of the asymptotically efficient positioning solution for a receiver, based on delay and Doppler measurements of known waveforms received from $K$ sources. To the best of our knowledge, this useful and general formula is new and represents one of the key result of this paper.

In the specific case of a GNSS receiver, and because of the huge distance between the receiver and the satellites, the angular velocities contained in $\boldsymbol{V}$ are always negligible compared with the normalized direction vectors included in $\boldsymbol{U}$. Hence, the matrix to be inverted in Eq. (28) can be usefully approximated by $\left[r \boldsymbol{U} \boldsymbol{U}^{T}\right]$ only, leading to a simpler 


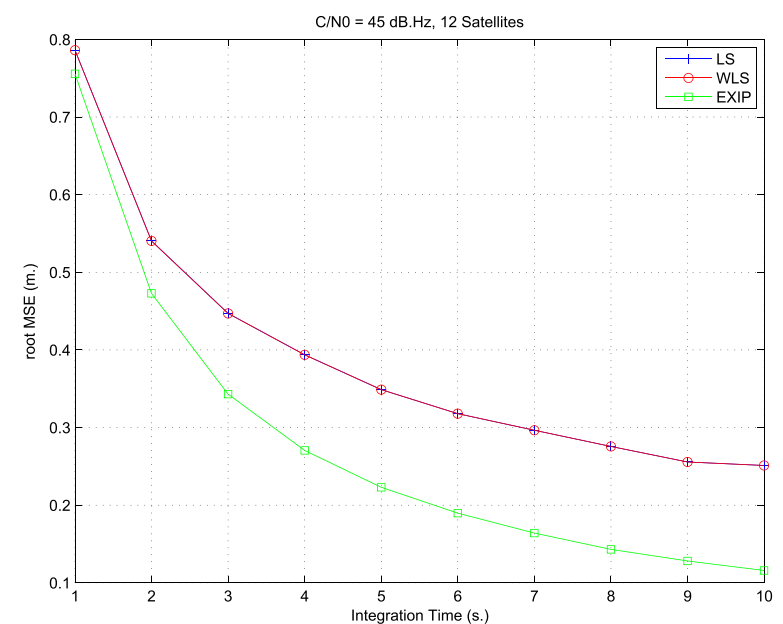

Fig. 1. Root Mean Square Positionning Error (m.) vs Integration Time, in open-sky.

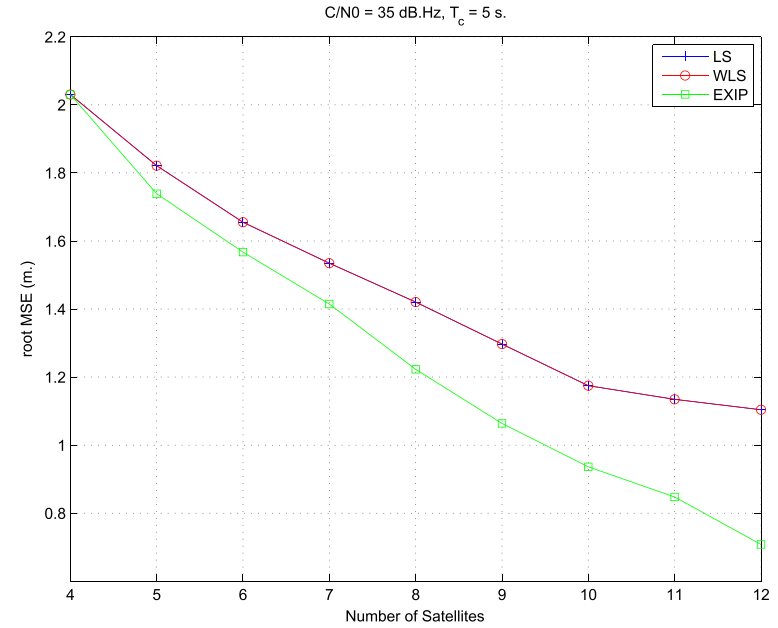

Fig. 2. Root Mean Square Positionning Error (m.) vs number of visible satellites.

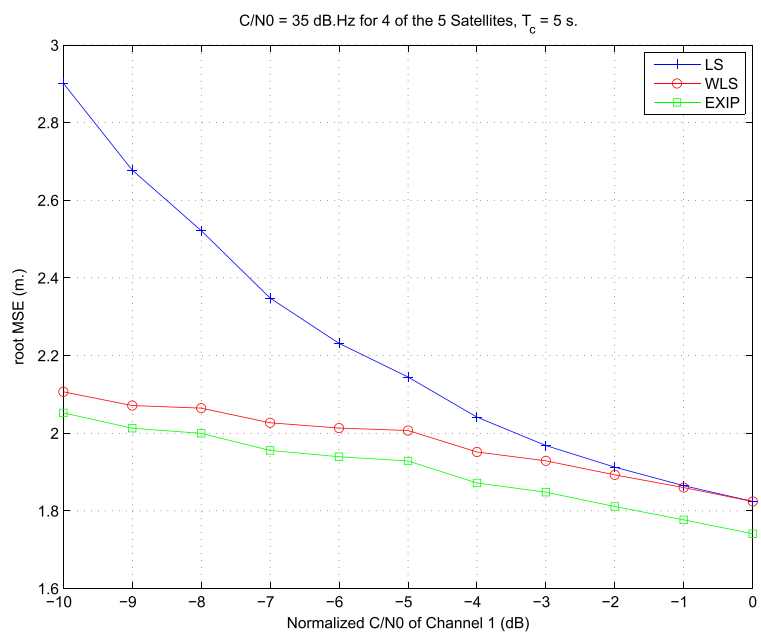

Fig. 3. Root Mean Square Positionning Error (m.) vs one channel fading.

mulation:

$\frac{1}{c}\left(\widehat{\boldsymbol{p}}_{E X I P}-\boldsymbol{p}_{0}\right) \simeq-\left[\boldsymbol{U} \boldsymbol{U}^{T}\right]^{-1} \boldsymbol{U} \boldsymbol{\Delta} \boldsymbol{\tau}-\left(\frac{f_{0}^{2} T_{c}^{2}}{B^{2}}\right)\left[\boldsymbol{U} \boldsymbol{U}^{T}\right]^{-1} \boldsymbol{V P} U_{U^{T}}^{\perp} \boldsymbol{\Delta} \boldsymbol{b}$

This last expression allows a more simple interpretation as we have isolated the contribution of the delay from the contribution of the Doppler:
- The first term, $-\left[\boldsymbol{U} \boldsymbol{U}^{T}\right]^{-1} \boldsymbol{U} \boldsymbol{\Delta} \boldsymbol{\tau}$, is shown to be (see Appendix B) exactly the classical WLS solution (where the weights are proportional to each channel received power), when considering only the pseudo-range measurements.

- The second term, $-\left(\frac{f_{0}^{2} T_{c}^{2}}{B^{2}}\right)\left[\boldsymbol{U} \boldsymbol{U}^{T}\right]^{-1} \boldsymbol{V} \boldsymbol{P}_{U^{T}}^{\perp} \boldsymbol{\Delta} \boldsymbol{b}$, is a correction that allows to improve the receiver position estimation when using the information included in the Doppler measurements. It has to be noticed that the influence of this correction is proportional to the square of the integration time $T_{c}$. Then, this correction is somehow negligible when dealing with short observation times, but becomes informative for longer ones.

Hence, when considering short integration times, the classical and widespread WLS procedure, based on the pseudo-ranges, is nearly optimal. But, as soon as one can access to longer integration times - say about one second for C/A GPS signals - (see Section 5 for more precise information), the corrective term should be taken into account to achieve a Doppler-aided asymptotically efficient solution. Indeed, the resolution of the ranging measurement is inversely proportional to the signal bandwidth whereas the angular resolution provided by the Doppler measurement is inversely proportional to the integration time, or in other words, inversely proportional to the synthetic antenna size due to the satellites motion. Thereby, the precision induced by the Doppler measurement increases much more rapidly than the precision linked to the delay. Using such qualitative considerations to give an order of magnitude, one roughly needs a $3 \mathrm{~s}$ integration time to obtain the same resolution than for a classical $1 \mathrm{MHz}$ bandwidth C/A GPS signal.

It can be noticed that increasing the coherent integration time is not an easy task for a GNSS receiver, but it appears, nowadays, as a promising way to manage difficult signal reception, such as urban canyons or indoor. The two main effects that limit integration time are known to be the oscillators instability and the unknown navigation bit transitions, but solutions appears to compensate for these drawbacks. This is particularly the case for quasi-static receivers, using AssistedGPS (AGPS), differential measurements and compensated oscillators [22,23].

Moreover, in [21], the authors have already highlighted the benefits provided by the use of the information included in the Doppler shifts to improve the receiver position estimation. In the present paper, we prove that this approach is asymptotically efficient providing the exact weights that should be used in order to achieve such an optimal processing.

It can also be noticed that the proposed 2-step procedure is equivalent to the DPE only if the received signal matches the assumed model. In case of signal mismatches (multipath, jamming,...) the benefits of using a direct optimization, namely the redundant information contained in the channels, is partly lost. The only link that can still be exploited is between the delay and Doppler of one channel, that contains both the user position information. This is thus another advantage of using the proposed Doppler-aided solution. Indeed, we can expect a corrective effect when the delay is biased whereas the Doppler remains correct. This is typically the case in a multipath environment, where the receiver is static (see Figs. 4 and 5).

Last, it can be noticed that the proposed algorithm does not require the estimation of the receiver clock delay and drift shifts. These unknowns have been eliminated in the receiver position and speed calculation but can be easily estimated using Eq. (19).

\section{Numerical illustrations}

The goal of this section is to asses the potential improvement of the proposed Doppler-aided WLS solution, named "EXIP" in the following figures, compared to the widespread one based only on the pseudoranges. This last solution is simply the first term of Eq. (29) and will be 


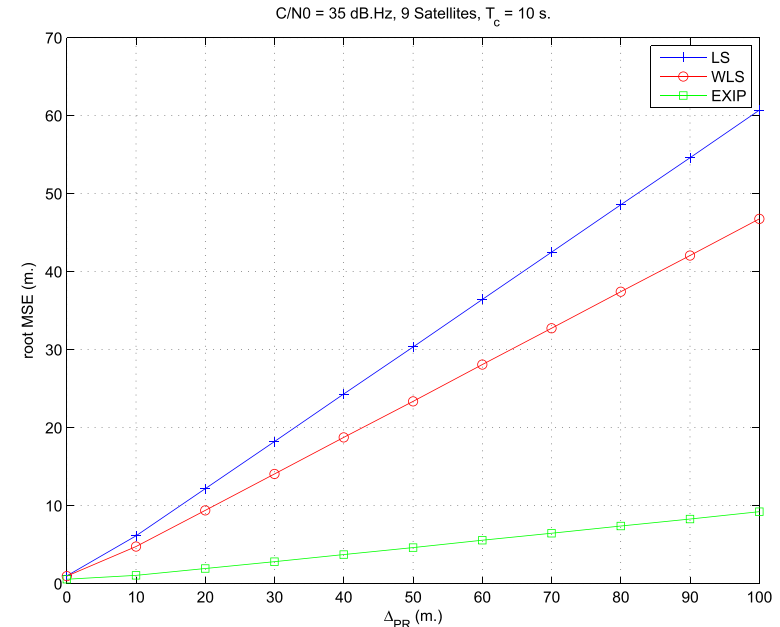

Fig. 4. Root Mean Square Positionning Error (m.) vs one channel multipath bias.

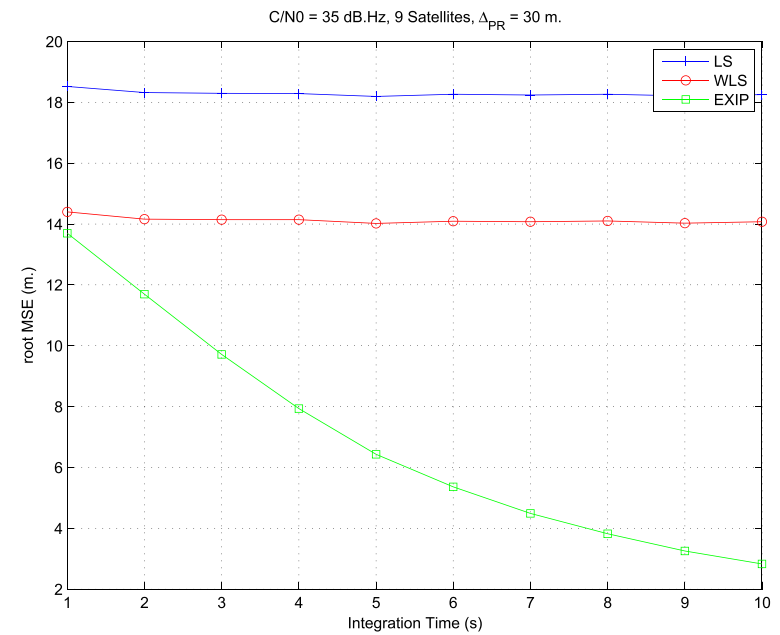

Fig. 5. Root Mean Square Positionning Error (m.) vs one channel multipath bias.

named "WLS". We have also added the standard LS solution for comparison, that assumes the same power $\left(P_{k}\right)$ for all channels. As stated in the previous section, the potential benefits of the proposed modified solution will only appear for long integration times. We compare the 3 above-mentioned solutions, through the root Mean Square Error (MSE) on the estimated position using Monte-Carlo simulations, on 3 different scenarios:

1. An open-sky scenario (Fig. 1) with 12 satellites $\left(C / N_{0}=45 \mathrm{~dB} \mathrm{~Hz}\right)$ where we vary the integration time to determine up to which observation time the classical WLS solution can be considered as optimal.

2. A more complicated scenario, where the number of visible satellites decreases (Fig. 2) and where one satellite is in constant fading during the whole integration time (Fig. 3). In this scenario we have chosen $C / N_{0}=35 \mathrm{~dB} \mathrm{~Hz}$.

3. A multipath scenario (Figs. 4 and 5) where the receiver is supposed static. In this case, a strong multipath (Signal to Multipath Ratio
( $S M R=3 \mathrm{~dB}$ ) biases the pseudo-range output of the correlator, whereas the Doppler of corresponding channel is still unbiased. We consider here 9 satellites in the field of view with $c$ / $N_{0}=35 \mathrm{~dB} \mathrm{~Hz}$.

We consider only the standard C/A $1 \mathrm{MHz}$ bandwidth signals.

From (Fig. 1), we can see that since the integration time is longer than a few seconds, the improvement due to the Doppler correction term cannot be ignored and can reach a division by 2 of the positioning error for a $10 \mathrm{~s}$ integration time. It can be noticed that in this simulation, all satellite signal powers are assumed to be equal so that the LS and WLS procedures are the same.

We now consider the influence of the number of satellites for a lower $C / N 0$ of $35 \mathrm{~dB} . \mathrm{Hz}$ and for an integration time of $T_{c}=5 \mathrm{~s}$., in Fig. 2. The gain provided by the proposed procedure becomes larger as the number of visible satellites grows. Once again, the different satellite signal powers have been chosen equal, leading to the same performance for the LS and WLS procedures.

Fig. 3 allows to analyse the behaviour of the 3 procedures in case of signal fading. In this simulation, we vary the fading of one of the 5 inview satellites. 4 signals are chosen to have a $C / N_{0}=35 \mathrm{~dB} \mathrm{~Hz}$ level and the last one changes from 25 to $35 \mathrm{~dB} \mathrm{~Hz}$. Obviously, in this case, we can see the superiority of the WLS compared to the classical LS, but the corrective Doppler term allows to improve, again, the precision in such a constrained scenario.

To finish with, we try out our proposed scheme in a multipath scenario. We consider that we have 9 satellites in the field of view of a static receiver, with the same $C / N_{0}=35 \mathrm{~dB} \mathrm{~Hz}$. One of the channels is in fading with a strong multipath so that the $\mathrm{C}=\mathrm{N} 0$ of the Line Of Site (LOS) and of the multipath are respectively $30 \mathrm{~dB} . \mathrm{Hz}$ and $32 \mathrm{~dB} . \mathrm{Hz}$. In this situation, the corresponding channel will have a pseudo-range bias whereas the Doppler frequency remains unbiased. In Fig. 4, we vary the relative delay between the multipath and the LOS signal, causing a pseudo-range bias. We can see that, in this case, the improvement due to the Doppler corrective term is more important. Indeed, the Doppler information remains correct whereas the pseudo-range one is biased. The corrective Doppler term tends to compensate for the pseudo-range biased information. This behaviour is emphasized on Fig. 5 where the integration time varies. We can clearly see that the more the integration time increases, the better the performance will be when using the proposed scheme, unlike the classical WLS algorithms.

\section{Conclusions}

This paper focused on the optimality of the positioning procedure for a GNSS receiver. We have shown that the classical WLS minimization, that only exploits the pseudo-ranges measurement is near efficient only when the integration time is short. Indeed, as soon as the observation time increases, the optimal algorithm consists in a Doppler corrected version of the widespread WLS algorithm. This modified WLS procedure is shown to be asymptotically equivalent, in a clear-sky environment, to the so called DPE, which results from the direct ML procedure. We have assessed the validity of this efficient Doppler-aided WLS procedure through numerical simulations and have shown that we can get a significant improvement in complicated scenarios.

\section{Appendix A}

As previously stated, all cross-correlation between different satellite signals are supposed to be approximatively and identically null for all relative delays and Doppler shifts, so that we can write down 
$R_{k, l}\left(\tau-\tau_{k}\right)=\int c_{k}\left(t-\tau_{k}\right) c_{l}(t-\tau) e^{-2 i \pi f_{0}\left(b_{k}-b_{l}\right) t} d t \simeq 0 \quad \forall \tau \quad$ if $k \neq l$

Hence, the first and second order derivatives will also be identically null if $k \neq l$ :

$0 \simeq \frac{\partial R_{k, l}\left(\tau-\tau_{k}\right)}{\partial \tau}=\int c_{k}\left(t-\tau_{k}\right) \frac{\partial c_{l}(t-\tau)}{\partial \tau} e^{-2 i \pi f_{0}\left(b_{k}-b_{l}\right) t} d t=-\int c_{k}\left(t-\tau_{k}\right) \frac{\partial c_{l}}{\partial t}(t-\tau) e^{-2 i \pi f_{0}\left(b_{k}-b_{l}\right) t} d t$

$0 \simeq \frac{\partial^{2} R_{k, l}\left(\tau-\tau_{k}\right)}{\partial \tau^{2}}=\int c_{k}\left(t-\tau_{k}\right) \frac{\partial^{2} c_{l}(t-\tau)}{\partial \tau^{2}} e^{-2 i \pi f_{0}\left(b_{k}-b_{l}\right) t} d t=\int c_{k}\left(t-\tau_{k}\right) \frac{\partial^{2} c_{l}}{\partial t}\left(t-\tau^{2}\right) e^{-2 i \pi f_{0}\left(b_{k}-b_{l}\right) t} d t$

The conversion to discrete time series shows that:

$\left(\partial \boldsymbol{c}_{k} \odot \boldsymbol{e}_{k}\right)^{H} \boldsymbol{a}_{l} \simeq 0 \quad$ if $k \neq l$

$\left(\partial^{2} \boldsymbol{c}_{k} \odot \boldsymbol{e}_{k}\right)^{H} \boldsymbol{a}_{l} \simeq 0$ if $k \neq l$

In the case of autocorrelation functions, we make use of the Parseval theorem:

$\int \frac{\partial c_{k}\left(t-\tau_{k}\right)}{\partial t} c_{k}\left(t-\tau_{k}\right) d t=\int(2 i \pi f) C_{k}(f) C_{k}^{*}(f) d f=(2 i \pi) \int f\left|C_{k}(f)\right|^{2} d f$

which is null because of the anti-symmetry of the function $f\left|C_{k}(f)\right|^{2}$.

Remark 1. We can remark that Eq. (A.6) represents the first derivative of the $k$-th signal autocorrelation function in its maximum as:

$\left.\frac{\partial R_{k}\left(\tau-\tau_{k}\right)}{\partial \tau}\right|_{\tau_{k}}=\left.\frac{\partial \int c_{k}\left(t-\tau_{k}\right) c_{k}(t-\tau) d t}{\partial \tau}\right|_{\tau_{k}}=\left.\int c_{k}\left(t-\tau_{k}\right) \frac{\partial c_{k}(t-\tau)}{\partial \tau} d t\right|_{\tau_{k}}=-\int c_{k}\left(t-\tau_{k}\right) \frac{\partial c_{k}}{\partial t}\left(t-\tau_{k}\right) d t$

which is actually null.If we do the same with the second order derivative of the autocorrelation function, we can show that

$\int \frac{\partial^{2} c_{k}\left(t-\tau_{k}\right)}{\partial t^{2}} c_{k}\left(t-\tau_{k}\right) d t=\int(2 i \pi f)^{2} C_{k}(f) C_{k}^{*}(f) d f=-(2 \pi)^{2} \int f^{2}\left|C_{k}(f)\right|^{2} d f=-(2 \pi)^{2} \Delta_{f}^{2} \int\left|C_{k}(f)\right|^{2} d f=-\left(2 \pi \Delta_{f}\right)^{2} \int\left|c_{k}(t)\right|^{2} d t=-\left(2 \pi \Delta_{f}\right)^{2} N T_{s}$

where we define the signal frequency span as $\Delta_{f}^{2}=\frac{\int f^{2}\left|C_{k}(f)\right|^{2} d f}{\int\left|C_{k}(f)\right|^{2} d f}$.

If considering a uniform power spectrum density for the signal of interest, the relationship between $\Delta_{f}$ and the bandwidth $B$ is shown to be $\Delta_{f}^{2}=\frac{B^{2}}{12}$.

With the same method, we can also evaluate:

$\int \frac{\partial c_{k}\left(t-\tau_{k}\right)}{\partial t} \frac{\partial c_{k}\left(t-\tau_{k}\right)}{\partial t} d t=-\int(2 i \pi f)^{2} C_{k}(f) C_{k}^{*}(f) d f=(2 \pi)^{2} \int f^{2}\left|C_{k}(f)\right|^{2} d f=\left(2 \pi \Delta_{f}\right)^{2} N T_{s}$

Now, re-writing Eq. (A.1) as

$0 \simeq R_{k, l}\left(f_{k}, u\right)=\int c_{k}(t) e^{-2 i \pi f_{k} t} c_{l}(t) e^{-2 i \pi u t} d t \quad$ if $k \neq l=\int c_{k}(t) e^{-2 i \pi f_{k} t}\left(c_{l}(t) e^{2 i \pi u t}\right)^{*} d t=\int C_{k}\left(f+f_{k}\right)\left(C_{l}(f-u)\right)^{*} d t$

that should be identically null for any frequency shift $u$, so that we have:

$0 \simeq \frac{\partial R_{k, l}\left(f_{k}, u\right)}{\partial u}=\int C_{k}\left(f+f_{k}\right) \frac{\partial C_{l}(f-u)^{*}}{\partial u} d t \quad$ if $k \neq l=-\int C_{k}\left(f+f_{k}\right)\left(\frac{\partial C_{l}}{\partial f}(f-u)\right)^{*} d t=-\int c_{k}(t) e^{-2 i \pi f_{k} t}\left((2 i \pi t) c_{l}(t) e^{-2 i \pi u t}\right)^{*} d t$

showing that $\left(\boldsymbol{c}_{k} \odot \boldsymbol{e}_{k}\right)^{H}\left(\boldsymbol{t} \odot \boldsymbol{c}_{l} \odot \boldsymbol{e}_{l}\right) \simeq 0$ if $k \neq l$.

Note that $\left(\boldsymbol{c}_{k} \odot \boldsymbol{e}_{k}\right)^{H}\left(\boldsymbol{t} \odot \boldsymbol{c}_{k} \odot \boldsymbol{e}_{k}\right)=\left(\boldsymbol{c}_{k}\right)^{T}\left(\boldsymbol{t} \odot \boldsymbol{c}_{k}\right)=\sum_{n=0}^{N-1} n T_{s}$.

Taking the second order derivative, we also have:

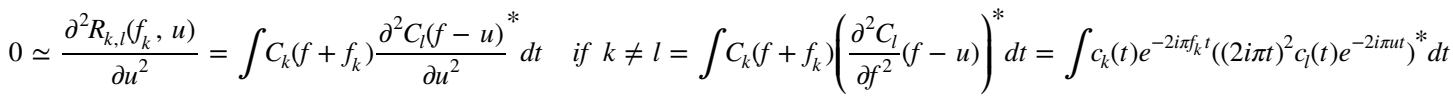

showing that $\left(\boldsymbol{c}_{k} \odot \boldsymbol{e}_{k}\right)^{H}\left(\boldsymbol{t}^{2} \odot \boldsymbol{c}_{l} \odot \boldsymbol{e}_{l}\right) \simeq 0$ if $k \neq l$.

Note that $\left(\boldsymbol{c}_{k} \odot \boldsymbol{e}_{k}\right)^{H}\left(\boldsymbol{t}^{\mathbf{2}} \odot \boldsymbol{c}_{k} \odot \boldsymbol{e}_{k}\right)=\left(\boldsymbol{c}_{k}\right)^{T}\left(\boldsymbol{t}^{\mathbf{2}} \odot \boldsymbol{c}_{k}\right)=\sum_{n=0}^{N-1}\left(n T_{s}\right)^{2}$.

To sum-up all these results:

$\boldsymbol{A}^{H} \boldsymbol{a}_{k}=N \boldsymbol{\delta}_{k}$

$\boldsymbol{A}^{H}\left(\partial \boldsymbol{c}_{k} \odot \boldsymbol{e}_{k}\right)=\mathbf{0}$

$\boldsymbol{A}^{H}\left(\partial^{2} \boldsymbol{c}_{k} \odot \boldsymbol{e}_{k}\right)=-4 \pi^{2} \Delta_{f}^{2} N \boldsymbol{\delta}_{k}$

$\left(\partial c_{k}\right)^{T}\left(\partial c_{k}\right)=4 \pi^{2} \Delta_{f}^{2} N$

$\boldsymbol{A}^{H}\left(\boldsymbol{t} \odot \boldsymbol{c}_{k} \odot \boldsymbol{e}_{k}\right)=\boldsymbol{\delta}_{k} \sum_{n=0}^{N-1} n T_{s}$ 
$\boldsymbol{A}^{H}\left(\boldsymbol{t}^{\mathbf{2}} \odot \boldsymbol{c}_{k} \odot \boldsymbol{e}_{k}\right)=\boldsymbol{\delta}_{k} \sum_{n=0}^{N-1}\left(n T_{s}\right)^{2}$

where $\boldsymbol{\delta}_{k}$ is the Kronecker vector composed of 0 expect at $k$-th-position, where it is 1 .

\section{Appendix B}

The second step of the classical two-stages procedure when considering only the delay measurements is based on the linearized Eq. (16).

$\Delta \tau_{r} \simeq-\frac{U_{0}^{T} \Delta p}{c}+\tau_{0} \mathbf{1}$

where $\boldsymbol{\Delta} \boldsymbol{\tau}_{r}(k)=\boldsymbol{\Delta} \boldsymbol{\eta}_{k}(1), \boldsymbol{U}=\left[\begin{array}{ll}\boldsymbol{u}_{\mathbf{0}_{0}} \cdots \boldsymbol{u}_{\boldsymbol{K}_{0}}\end{array}\right]$ and $\mathbf{1}=\left[\begin{array}{lll}1 & 1 & \cdots\end{array}\right]^{T}$. The weighted least square to be minimized writes as follows:

$C\left(\boldsymbol{\Delta} \boldsymbol{p}, \tau_{0}\right)=\left[\boldsymbol{\Delta} \boldsymbol{\tau}_{r}+\frac{\boldsymbol{U}_{0}^{T} \boldsymbol{\Delta} \boldsymbol{p}}{c}-\tau_{0} \mathbf{1}\right]^{T} \boldsymbol{W}\left[\boldsymbol{\Delta} \boldsymbol{\tau}_{r}+\frac{\boldsymbol{U}_{0}^{T} \boldsymbol{\Delta} \boldsymbol{p}}{c}-\tau_{0} \mathbf{1}\right]$

where $\boldsymbol{W}=\operatorname{diag}\left(\left[P_{0} \cdots P_{K-1}\right]\right)$.

Deriving with respect to the receiver clock bias, $\tau_{0}$, conducts to:

$\widehat{\tau}_{0}=\frac{\boldsymbol{1}^{T} \boldsymbol{W}\left(\boldsymbol{\Delta} \boldsymbol{\tau}_{r}+\frac{\boldsymbol{U}_{0}^{T} \Delta p}{c}\right)}{\operatorname{Tr}\{\boldsymbol{W}\}}=\overline{\Delta \tau}+\overline{\boldsymbol{u}}^{-T} \frac{\boldsymbol{\Delta} \boldsymbol{p}}{c}$

Inserting this expression in Eq. (B.2) leads to:

$\widetilde{C}(\Delta p)=\left[\left(\Delta \tau_{r}-\overline{\Delta \tau} \mathbf{1}\right)+\left(U_{0}-\bar{u} \mathbf{1}^{T}\right)^{T} \frac{\Delta p}{c}\right]^{T} W\left[\left(\Delta \tau_{r}-\overline{\Delta \tau} \mathbf{1}\right)+\left(U_{\mathbf{0}}-\bar{u} \mathbf{1}^{T}\right)^{T} \frac{\Delta p}{c}\right]$

which minimum in obtained for:

$\frac{\widehat{\boldsymbol{p}}}{c}=-\left[\boldsymbol{U} \boldsymbol{U}^{T}\right]^{-1} \boldsymbol{U} \boldsymbol{\Delta} \boldsymbol{\tau}$

\section{References}

[1] S. Bancroft, An algebraic solution of the GPS equations, IEEE Trans. Aerosp. Electron. Syst. 21 (7) (1985) 56-69.

[2] P. Closas, C.F.-P.J.A. Fernandez-Rubio, Direct position estimation approach outperforms conventional two-steps positioning, in: Proceedings of the 17th European Signal Processing Conference (EUSIPCO 2009), 2009, pp. 1958-1962.

[3] P. Closas, C. Fernandez-Prades, J.A. Fernandez-Rubio, Cramer-Rao bound analysis of positioning approaches in GNSS receivers, IEEE Trans. Signal Process. 57 (10) (2009) 3775-3786.

[4] P. Closas, C. Fernandez-Prades, J.A. Fernandez-Rubio, Maximum likelihood estimation of position in GNSS, IEEE Signal Process. Lett. 14 (5) (2007) 359-362.

[5] P. Closas, C. Fernandez-Prades, J.A. Fernandez-Rubio, A. Ramirez-Gonzalez, On the maximum likelihood estimation of position, in: Proceedings of ION GNSS, 2006, pp. 1800-1810.

[6] P. Closas, C. Fernandez-Prades, D. Bernal, J.A. Fernandez-Rubio, Bayesian direct position estimation, in: Proceedings of ION GNSS, 2008, pp. 183-190.

[7] P. Closas, C. Fernandez-Prades, Bayesian nonlinear filters for direct position estimation, in: Proceedings of the IEEE Aerospace Conference, 2010, pp. 1-12.

[8] A.P. Dempster, N.M. Laird, D.B. Rubin, Maximum likelihood from incomplete data via the EM algorithm, J. R. Stat. Soc. Ser. B 39 (1) (1977) 1-38.

[9] F. Antreich, J.A. Nossek, W. Utschick, Maximum likelihood delay estimation in a navigation receiver for aeronautical applications, Elsevier Aerosp. Sci. Technol. 12 (3) (2008) 256-267.

[10] S. Rougerie, G. Carrie, F. Vincent, L. Ries, M. Monnerat, A new multipath mitigation method for GNSS receivers based on antenna array, Int. J. Navig. Observ., (special issue "Advances in Signal Tracking for GNSS Receivers: Theory and Implementation").

[11] P. Stoica, T. Soderstrom, On reparametrization of loss functions used in estimation and the invariance principle, Signal Process. 17 (4) (1989) 383-387.

[12] A.L. Swindlehurst, P. Stoica, Maximum likelihood methods in radar array signal processing, Proc. IEEE 86 (2) (1998) 421-441.

[13] F. Vincent, O. Besson, Estimating time-varying DOA and doppler shift in radar array processing, IEE Proc. Radar Sonar Navig. 147 (6) (2000) 285-290.

[14] F. Antreich, J.A. Nossek, G. Seco-Granados, A.L. Swindlehurst, The extended invariance principle for signal parameter estimation in an unknown spatial field, IEEE Trans. Signal Process. 59 (7) (2011) 3213-3225.

[15] A. Amar, A.J. Weiss, New asymptotic results on two fundamental approaches to mobile terminal location, in: Proceedings of ISCCSP, 2008, pp. 1320-1323.

[16] A. Amar, A.J. Weiss, Localization of narrowband radio emitters based on doppler frequency shifts, IEEE Trans. Signal Process. 56 (11) (2008) 5500-5508.

[17] A.J. Weiss, Direct position determination of narrowband radio frequency transmitters, IEEE Signal Process. Lett. 11 (5) (2004) 513-516.

[18] A.J. Weiss, A. Amar, Direct position determination of multiple radio signals, EURASIP J. Appl. Signal Process. (2005) 37-49.

[19] A.J.V. Dierendonck, P. Fenton, T. Ford, Theory and performance of narrow correlator spacing in a GPS receiver, Navig.: J. Inst. Navig. 39 (3) (1992) 265-283.

[20] R.D.J.V. Nee, Spread-spectrum code and carrier synchronization errors caused by multipath and interference, IEEE Trans. Aerosp. Electron. Syst. 29 (4) (1993) $1359-1365$.

[21] L. Li, J. Zhong, M. Zhao, Doppler-aided GNSS position estimation with weighted least squares, IEEE Trans. Veh. Technol. 60 (8) (2011) 3615-3624.

[22] A. Broumandan, J. Nielsen, G. Lachapelle, Enhanced detection performance of indoor GNSS signals based on synthetic aperture, IEEE Trans. Veh. Technol. 59 (6) (2010) 2711-2724.

[23] A. Broumandan, J. Nielsen, G. Lachapelle, Indoor GNSS signal acquisition performance using a synthetic antenna array, IEEE Trans. Aerosp. Electron. Syst. 47 (2) (2011) 1337-1350. 